\title{
EL DESARROLLO DEL COMERCIO INTERNACIONAL DEL PUERTO DE PUNTA ARENAS (CHILE), 1905-1914
}

RICARDO SANTANA F. ${ }^{\mathrm{a}}$

\section{RESUMEN}

El artículo analiza el desarrollo comercial con datos cuantitativos obtenidos de los Anuarios Comerciales de las República de Chile para observar en detalle el grado comercial que adquirió el puerto de Punta Arenas entre los años de mayor auge económico de la región, también conocido como la época dorada. Para esto, se hace una introducción del período de la colonia y de la expansión que tuvo la ciudad de Punta Arenas a finales del siglo XIX para luego pasar a explicar cómo el comercio internacional fue un pilar fundamental para este puerto de la zona austral.

PALABRAS CLAVE: Comercio internacional, Punta Arenas, economía, exportación, importación.

\section{THE INTERNATIONAL TRADE DEVELOPMENT OF THE PUNTA ARENAS SEA PORT (CHILE), 1905-1914}

\begin{abstract}
This article analyzes the commercial development of the Punta Arenas se port using quantitative data obtained from the yearbooks of the Republic of Chile. The aim is to observe in detail the level of commercial activity in the port of Punta Arenas during the years of greater economic boom in the region, known as the golden age. An overview of the colony's period and the expansion of the city of Punta Arenas at the end of the 19th century is provided to address this objective. Subsequently, the essential role of the international trade for this port in the southernmost zone of Chile is illustrated and interpreted.
\end{abstract}

KEY WORDS: International trade, Punta Arenas, economy, export, import.

a Investigador Adjunto, Centro de Estudios Latinoamericanos sobre China (CELC). Facultad de Humanidades y Ciencias Sociales de la Universidad Andrés Bello. rsantanafriedli@gmail.com 


\section{ANTECEDENTES HISTÓRICOS DE PUNTA ARENAS COMO NÚCLEO DE DESARROLLO EN MAGALLANES}

\section{Introducción}

Aplicando un nuevo enfoque cuantitativo a la historiografía regional, este trabajo pretende ofrecer una mirada detallada y de mayor calidad de la importancia comercial que significó el puerto de Punta Arenas para la zona austral. Mediante el trabajo con fuentes primarias, tanto de archivos públicos como privados, la investigación recolecta información que no había sido consultada por otros estudios que abarcan ya de por sí un período ampliamente abordado y catalogado como de auge. De esta manera, el objetivo principal es lograr con la mayor calidad posible, el enriquecimiento del conocimiento desde una perspectiva estadística del comercial del período.

Principalmente los datos fueron extraídos desde los Anuarios Comerciales de la República de Chile, los cuales se encontraban en formato de microficha en la Biblioteca Nacional de Chile en Santiago. No obstante, también se realizó un trabajo minucioso con la prensa El Magallanes, la cual fue consultada para recopilar el tonelaje de entradasalida del puerto y el número de embarcaciones que frecuentaban el puerto de Punta Arenas. Se tuvo acceso a originales de El Magallanes en la biblioteca privada de La Prensa Austral y en la biblioteca del Instituto de la Patagonia, ambos en la ciudad de Punta Arenas. Algunas copias entre los años de 1912 al 1914 del diario fueron consultados en la Biblioteca Nacional de Chile en formato de microficha para corroborar algunos datos del tráfico marítimo. Asimismo, el Censo General de la República de Magallanes también fue de gran aporte estadístico para conocer la realidad del puerto en los años previos al período abarcado por el estudio y como fuente para cotejar datos del año 1906.

\section{Época de colonización y expansión de Magallanes}

Por la importancia geopolítica de la región de Magallanes, Bernardo O'Higgins siempre estuvo empeñado en emprender una colonización del Estrecho de Magallanes. Una de sus últimas preocupaciones en su exilio en Perú, fue recordar mediante correspondencia al Presidente Bulnes la importancia de colonizar esa zona austral (Martinic, 2006a).

Es así que a mediados de 1842, O'Higgins es informado de la organización de una expedición colonizadora, encargada por el Intendente de Chiloé Domingo Espiñeira y dirigida por el capitán Juan Williams. La expedición había recalado hondo en el alma del prócer, tanto así que en su lecho de muerte las última palabras serían en el recuerdo de esa tierra "Magallanes, Magallanes..."

La expedición partió el 21 de mayo de 1843 desde el puerto de Ancud, travesía que luego de cuatro meses desembocaría en la bahía de San Juan y donde se instalaría el asentamiento de Fuerte Bulnes el 30 de octubre.

A pesar de contar la zona aledaña de los recursos esenciales como agua dulce, madera y refugio contra las inclemencias del clima, no se disponía de amplios campos de pastoreo y la tierra era muy rocosa para el cultivo. Por lo que luego de las penurias, el gobernador José de los Santos Mardones desplazó la colonia hacia la ribera del río del Carbón para fundar la nueva población en Punta Arenosa.

Punta Arenosa (conocida posteriormente como Punta Arenas) al ser un paso obligatorio para desplazarse entre el Pacífico y el Atlántico, logra posicionarse incipientemente como un importante centro urbano en el extremo austral gracias al comercio y tráfico internacional. Esto permitió a la población adquirir toda clase de artículos como relata el gobernador Oscar Viel en una de sus cartas a su hermano Benjamín:

La colonia sigue prosperando i te aseguro da gusto ver que todos aquellos artículos que antes valían un dineral, en el día se consiguen por lo menos lo mismo que en Valparaíso. Pues con los vapores todo viene de Europa i esta ganara cada día más pues desde Agosto, los vapores tanto de Europa como de Valparaíso. Serán quincenales (Viel, 1870).

El desarrollo del tráfico y comercio internacional por la zona austral, hubiera tenido un 
mayor impacto en el nuevo establecimiento sino fuera por el fin del período aurífero en el Estado de California, que ya para 1855 se había dado por finalizado, disminuyendo las recaladas a Punta Arenas (Bascuñán, 2013).

El puerto de Punta Arenas adquiriría mayor relevancia, cuando en 1868 la Pacific Steam Navigation Company informa que su navegación hacia el puerto de Valparaíso desde Europa dispondría de una nueva recalada: Punta Arenas. Un año después la Compañía Alemana de Vapores Kosmos regularizaba su tráfico por el estrecho a través del mismo puerto. Así la colonia fue adquiriendo una mayor presencia internacional, la cual luego cobró un mayor impulsada por el descubrimiento de oro en el río de las Minas en 1869, el cual trajo una mayor presencia de inmigrantes europeos a la región.

La firma del Tratado de Límites entre Chile y Argentina de 1881 zanjó la controversia territorial con el país vecino, permitiendo alcanzar la estabilidad necesaria para la concesión de terrenos sobre el territorio patagónico chileno. De esta forma, a través del gobernador Oscar Viel, se concedieron primero los campos aledaños a la ciudad de Punta Arenas para labores agrícolas y ganaderas, esto aprovechando las tierras para el pastoreo de ganado bovino y ovino. Esto llevó a iniciar un plan de colonización con inmigrantes europeos para otorgar pasaje libre en tercera clase, y la entrega de una finca de 48 hectáreas por cada familia y 12 hectáreas más por cada hijo mayor de 14 años y otros beneficios.

La presencia de colonos europeos permitió un nuevo impulso en el desarrollo de una economía ovina, inspirada en el éxito logrado por los británicos en las islas Falkland y por el emprendimiento de Enrique Reynard (Martinic, 2006a), lo cual motivó a otros para adquirir nuevas tierras hacia el interior del territorio.

\section{Época dorada de Magallanes}

Hacia mediados de la primera década del siglo XX la expansión territorial en la región de Magallanes había terminado, pero el desarrollo demográfico todavía estaba en curso gracias a la nueva migración de más europeos y de nacionales procedentes del centro y sur del país (Martinic, 2002a). Durante esta década la actividad ganadera se había convertido en la principal actividad económica, la que tenía la característica de impulsar el desarrollo de otras áreas dentro de la región como el comercio. A partir del año 1905, la producción ganadera se vería potenciada aún más por la instalación del primer frigorífico que ofrecería un importante elemento de valor agregado y la diversificación de los productos derivados del mismo sector económico. El desarrollo del primer frigorífico no hubiera sido posible sin los lazos ingleses, que en la región ya llevaba un tiempo, los cuales habían permitido iniciar la exportación de la producción ganadera hacia el Reino Unido. Así es como por iniciativa de Mauricio Braun, uno de los mayores emprendedores de la región y un grupo de empresarios ingleses, entre ellos la Houlder Brothers de Londres, propusieron la instalación en 1903 de la primera planta de faenamiento y frigorización con la más alta tecnología conocida en la localidad de Río Seco, a las afuera de Punta Arenas (Martinic, 2002b). Para ello se efectuó la constitución de la The South American Export Syndicate Limited con sede en Londres y con un capital inicial de 30 mil libra esterlinas. Esta planta contaría con las maquinarias, instalaciones, tecnología de conservación de carnes y demás para el apropiado desarrollo de la industria.

Luego y gracias a los buenos resultados del primer frigorífico, el empresario regional José Menéndez quien con la The Patagonian Sheep Farming Co. y la Fred Waldron y otros, constituyeron la Compañía Frigorífica de Patagonia, con un capital de 100.000 libras esterlinas para la instalación del frigorífico en 1908 en Puerto Sara, bahía de San Gregorio (Martinic, 2002a). Este último iniciaría sus faenas con una producción de 145 mil capones congelados. La nueva planta amplió la industrialización de la carne ovina y derivados, permitiendo así el procesamiento de subproductos como sesos, lenguas, hígados, riñones, corazones mediante la conserva, diversificando de esta forma el negocio agroindustrial. Asimismo, la caza de ballena significó otra industria con un alto grado de tecnificación, la cual fue liderada por la Sociedad Ballenera de Magallanes a principios de 1906 y en la cual participó como presidente Mauricio Braun. Esta industria que inició sus actividades con instalaciones con maquinarias al sur de la península 
Brunswick, contó con al principio con el vapor Almirante Montt y luego paulatinamente fueron incorporándose nuevas embarcaciones hasta llegar a un total de cinco vapores cazadores y buquesfábrica (Martinic, 1995).

\section{EL COMERCIO INTERNACIONAL COMO PILAR DE LA ECONOMÍA REGIONAL}

\section{Crecimiento del comercio internacional de Punta Arenas}

La apertura hacia los mercados europeos de productos principalmente provenientes de la industria ganadera, produjeron un fuerte impulso en el comercio internacional de la región. Gracias a esto, el puerto logró posicionarse como el principal centro urbano al sur del paralelo 46, zona que integra las regiones del sur de Chile, Tierra del Fuego y Santa Cruz (Ciselli, 1999). Como consecuencia, la emergencia del poblado a orillas del estrecho permitió no tan solo un acceso directo a bienes que difícilmente eran conseguidos por la falencia en la conectividad con los centros más pujantes de la época, sino que también facilitó que otros asentamientos australes lo tuvieran gracias a su vínculo con Punta Arenas.

Las facilidades comerciales $y$ de infraestructura del puerto chileno, no tan solo ofrecieron mejores oportunidades para salida de la producción nacional, sino que también el puerto era ampliamente utilizado por empresarios argentinos provenientes de diferentes localidades australes para la exportación e importación. En consecuencia, muchos establecimientos artesanales $e$ industriales instalaron en Punta Arenas sus casas matrices, las cuales ya para 1906 el 88\% estaba compuesto por inversionistas europeos y un $90 \%$ del comercio (Navarro, 1906).

La presencia de europeos que amasaron sus fortunas tras diferentes actividades económicas en la región, facilitó el contacto comercial con los mercados internacionales (principalmente del viejo mundo) gracias a sus anteriores vínculos con sus países de origen y por la afinidad cultural. Su desarrollo económico impulsó a toda la sociedad magallánica que se volcó hacia el comercio con Europa, tanto así que sobrepasaría al mismo movimiento marítimo regional y nacional. Como se observa en la Tabla 1, a partir de 1907 el tráfico marítimo fue liderado por barcos británicos y alemanes, quienes usaban como centro de negocios el puerto de Punta Arenas y como recalada en el paso hacia los puertos de Valparaíso y otras zonas del Pacífico.

La hegemonía que ostentaba el Imperio Británico y la batalla por la supremacía comercial con el Imperio Alemán también se reflejó durante todo el período de esplendor regional. Según los registros, durante el período entre 1906 y 1912 existió un claro dominio del tráfico de embarcaciones con bandera inglesa, seguido por las de origen alemán que nunca superaron al movimiento marítimo inglés. Mientras que las embarcaciones norteamericanas, las terceras en mayor cantidad entre las extranjeras, tuvieron una declinación en paso por el puerto a partir de 1909 a 1912.

Además del registro y cantidad de embarcaciones que utilizaban el puerto de Punta Arenas, se dispone gracias a los datos entregados por la Gobernación Marítima a la prensa, del peso en toneladas de cada embarcación que atracó. A través de este dato se ha podido armar el total del tonelaje que lo componían las embarcaciones, relevante indicador para medir la importancia real del tráfico en materia de transporte de carga para cada año. Estos datos completan la serie iniciada por Lautaro Navarro en su Censo General para el Territorio Magallánico, en el cual se observa el aumento de embarcaciones que transitaban por el estrecho de Magallanes desde 1868 a 1906.

Punta Arenosa pasó de mantener un flujo de apenas 78 embarcaciones en 1871 a 195 en 1879 , esto en parte gracias a los buenos resultados de la aclimatación de la oveja malvineras en territorio magallánico, lo que impulsó el tráfico de cabotaje por la demanda de madera y otras variedades de cargamentos para los incipientes establecimientos pastoriles (Martinic, 1977). Ya para inicios de siglo el número de embarcaciones que surcaban las aguas del estrecho había ascendido arriba de 700, apoyada por el esfuerzo de la compañía armadora "Braun \& Blanchard" que mantenía las líneas de transporte que unían el Pacífico con el Atlántico.

$\mathrm{Al}$ igual que el registro del número de embarcaciones (Fig. 1), el tonelaje por cabotaje exterior desde y hacia el puerto de Punta Arenas 
Tabla 1. Cantidad de embarcaciones de las cuatro principales nacionalidades que utilizaban el puerto de Punta Arenas entre 1906 y 1912, tanto de salida como de entrada. Fuente: Elaboración propia sobre la base de los datos estadísticos de los Anuarios Comerciales de la República de Chile.

\begin{tabular}{|c|c|c|c|c|c|c|c|c|c|c|}
\hline \multirow{2}{*}{$\begin{array}{l}\text { Punta Arenas } \\
\text { Nacionalidad }\end{array}$} & \multicolumn{2}{|c|}{1906} & \multicolumn{2}{|c|}{1907} & \multicolumn{2}{|c|}{1908} & \multicolumn{2}{|c|}{1909} & \multicolumn{2}{|c|}{1910} \\
\hline & Entrada & Salida & Entrada & Salida & Entrada & Salida & Entrada & Salida & Entrada & Salida \\
\hline Chilena & 395 & 399 & 81 & 86 & 93 & 87 & 116 & 116 & 119 & 128 \\
\hline Inglesa & 172 & 170 & 195 & 199 & 200 & 196 & 208 & 207 & 233 & 243 \\
\hline Alemana & 110 & 118 & 132 & 133 & 150 & 150 & 129 & 128 & 167 & 162 \\
\hline \multirow[t]{7}{*}{ Norteamericana } & 15 & 15 & 7 & 8 & 15 & 15 & 8 & 7 & 9 & 10 \\
\hline & \multicolumn{3}{|c|}{ Punta Arenas } & \multicolumn{2}{|c|}{1911} & \multicolumn{3}{|c|}{1912} & & \\
\hline & \multicolumn{3}{|c|}{ Nacionalidad } & Entrada & Salida & Entrada & \multicolumn{2}{|c|}{ Salida } & & \\
\hline & \multicolumn{3}{|c|}{ Chilena } & 186 & 126 & 198 & \multicolumn{2}{|l|}{135} & & \\
\hline & \multicolumn{3}{|c|}{ Inglesa } & 240 & 228 & 211 & \multicolumn{2}{|l|}{196} & & \\
\hline & \multicolumn{3}{|c|}{ Alemana } & 174 & 171 & 184 & 183 & & & \\
\hline & \multicolumn{3}{|c|}{ Norteamericana } & 7 & 7 & 4 & 3 & & & \\
\hline
\end{tabular}

experimentó un aumento que solamente fue interrumpido levemente en 1908 tras una baja a 437 mil toneladas de salida, pero que repuntó a 752 mil al siguiente año. Esta variación experimentada en 1908 pudo haber sido efecto de la creación de la "Sociedad Anónima Importadora y Exportadora de la Patagonia" en ese mismo año y que fusionó los intereses de las casas Braun \&
Blanchard y José Menéndez. La sociedad más conocida como "La Anónima", se convirtió en la más importante empresa de toda la Patagonia y generó un desequilibrio en las relaciones con las demás empresas mercantiles de Magallanes, provocando un año después la constitución de la "Sociedad Argentina del Sud"1. Estas dos firmas competirían por más de sesenta años en este

\section{- Tonelaje de Entrada $\quad$ Tonelaje de Salida}

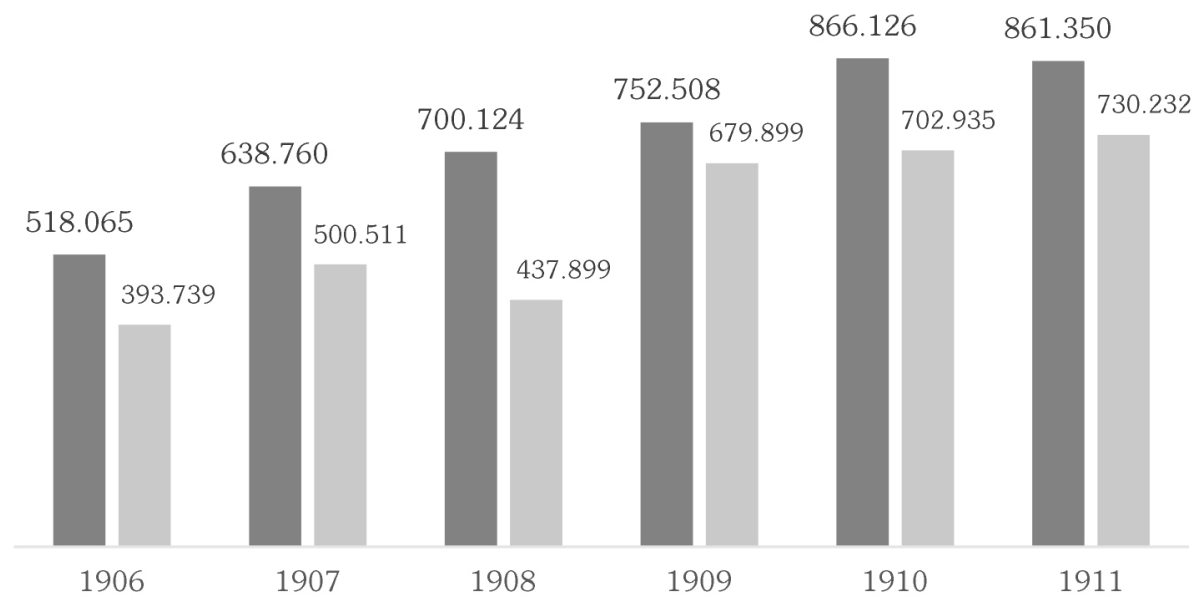

Fig. 1. Total anual del tonelaje exterior de entrada y salida del puerto de Punta Arenas entre 1906 y 1911. Fuente: Elaboración propia sobre la base de los datos estadísticos de los Anuarios Comerciales de la República de Chile. 
extremo sur del continente.

Gracias al desarrollo económico obtenido en las últimas dos décadas, Punta Arenas mantuvo un activo comercio exterior conEuropa, principalmente de productos derivados de la ganadería y la caza de ballenas. Magallanes contaba con una economía que favorecía a la demanda interna británica, la cual requería fuertemente de alimentos y de lana para su poderosa industria. Por esta razón, las exportaciones se enfocaron en la venta de lana de oveja la cual era producida en grandes cantidades, tanto en la Patagonia argentina como chilena. Sin embargo la instalación del primer frigorífico en 1905 significó para el comercio exterior regional la diversificación de la producción exportable, ofreciendo nuevas oportunidades de negocios, ya que era posible el envío de alimentos congelados a los mercados globales más importantes, sin que se viera en riesgo su preservación.

Como observamos en la Tabla 2, las exportaciones de los principales productos elaborados en la región experimentaron un crecimiento que se extendió hasta 1912, año en que posiblemente el efecto aduana y la huelga general pudieron incidir en la producción regional (con excepción de la exportación de aceite de ballena que no se vio afectado por la caída de 1912). Posteriormente los años 1913 y 1914 volvieron a crecer las exportaciones, manteniendo inclusive niveles mayores al período previo a 1912 .

El importante comercio de exportación era equilibrado con un importante comercio de importación, como se observa en la Tabla 3. Fue en 1911, un año antes de la instalación de la aduana, cuando se alcanzó el mayor valor de importación con casi diez millones y medio de pesos. Esta balanza comercial estaba centrada básicamente en la exportación de materias primas por la llegada de productos de procedencia animal, vegetal y bienes de mayor valor agregado que posiblemente la producción nacional no alcanzaba como bebidas, textiles y fármacos.

Desde la llegada de los primeros colonos a esta región del extremo sur de América, uno de los principales desafíos fue mantener un suministro constante y variado de alimentos para su alimentación. La instalación de los primeros cultivos de hortalizas y legumbres resistentes a las

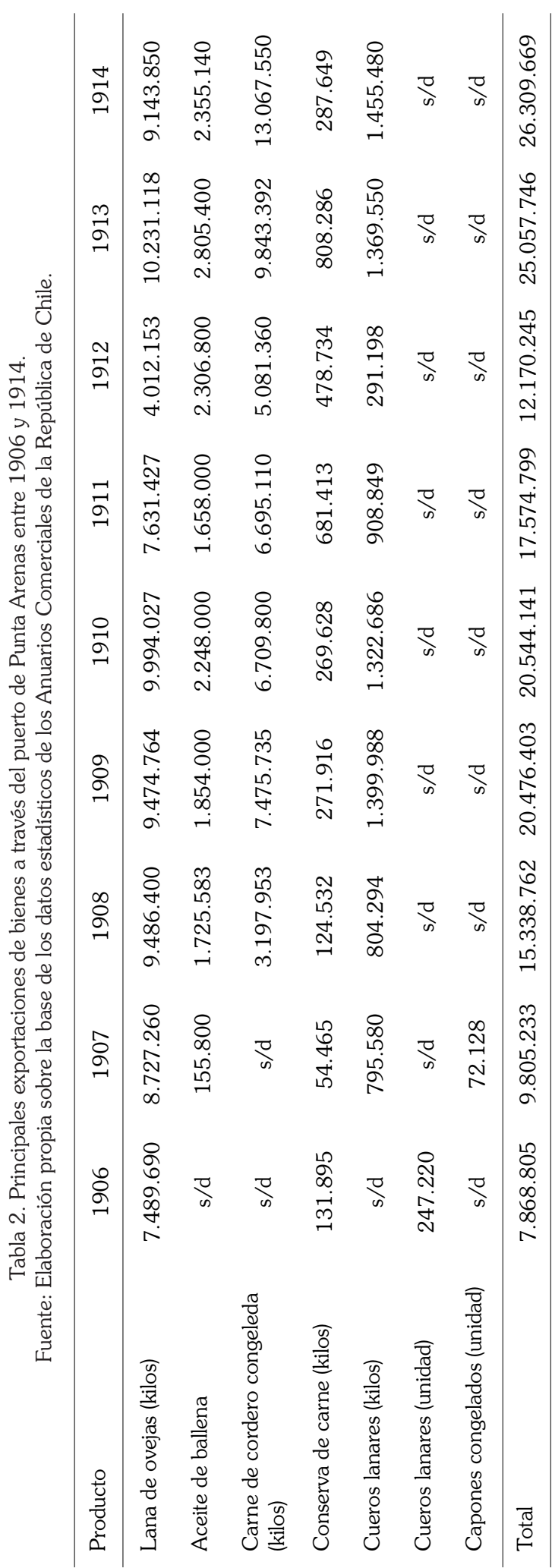


condiciones del clima ayudaron en el inicio de la próspera colonia, pero con el crecimiento de la población y el desarrollo económico alcanzado, la sociedad fue demandando una mayor variedad de productos que para adquirirlos era necesario comprarlos en otros mercados. Las clases más acomodadas y luego como consecuencia del desarrollo regional, una clase media con un mayor poder adquisitivo, fueron demandando nuevos productos o una mayor variedad alimentaria $y$ de bienes de diferente clase que pronto fueron llegando a la región.

El principal alimento en la dieta magallánica, la carne de ovino, empezó a ser acompañada de productos extranjeros de origen animal y vegetal, convirtiéndose en las principales categorías importadas. Asimismo, maquinas, herramientas, textiles y manufacturas, como también minerales fueron muy cotizados en el mercado regional, los cuales podían acceder sin mayores problemas ni contratiempos gracias a la calidad de puerto libre que ostentaba Punta Arenas. Sin embargo, el mayor producto importado fueron las bebidas $y$ licores, que inclusive llegaron a superar en valor de importación a los productos básicos requeridos en los hogares magallánicos.

Como se observa en la Tabla 3, durante el período entre 1907 y 1912 la categoría "Bebidas y Licores" se mantuvo como el principal producto importado al puerto, superando ampliamente a otros productos como los de origen animal o vegetal, los que se encuentran como los segundos productos más importados. Mientras que en los años 1913 y 1914 el valor importado sufrió una disminución drástica, afectada por la instalación de la aduana en Punta Arenas, acontecimiento que será tratado más adelante.

Como ya se mencionó, la amplia presencia europea en la sociedad magallánica, y gracias a la importancia del estrecho de Magallanes como paso obligatorio para embarcaciones que transitaban en ambos océanos, facilitó la llegada de gran cantidad de productos, los cuales superarian desproporcionadamente a los bienes nacionales. En consecuencia, ciertos sectores empresariales de la zona centro-sur de Chile mostraron su rechazo a la presencia y competencia del mercado patagónico. Es así que desde finales del siglo XIX y principios del XX a través del lobby impulsarian un movimiento para la eliminación de la categoría de puerto libre de Punta Arenas y frenar la presencia del comercio extranjero.

El censo de 1906 es una interesante fuente para el análisis de la presencia de los productos nacionales en la región de Magallanes. Como se observa en la Tabla 4, la presencia de productos provenientes de la agricultura, industria del calzado y licores eran los que mayor apertura tenían en el mercado austral. De esta forma, no es de extrañar que los grandes latifundistas de la zona central de Chile vieran a esta región como un nicho de mercado prometedor todavía por explorar y listo para la apertura de nuevos productos nacionales.

Fin del período de esplendor del comercio internacional de Punta Arenas

Luego de experimentar un notable período de crecimiento, la región entraría en un ocaso hacia principios de la segunda década del siglo $\mathrm{XX}$, producto de sucesos externos e internos que hicieron perder la particularidad y relevancia que ostentaba en la zona austral el puerto de Punta Arenas.

\section{Ley de cabotaje argentino}

El primer signo del ocaso provendría desde la legislación argentina con la instalación de la ley de cabotaje en 1910. La ley impulsada por el gobierno central de Buenos Aires y apoyada por los armadores bonaerenses tenía dos objetivos: impulsar la navegación con bandera argentina en el Delta del Río de la Plata por la fuerte presencia de embarcaciones extranjeras y expandir la influencia argentina de embarcaciones nacionales hacia los puertos del sur del país mediante la reserva del tráfico a embarcaciones con matrícula argentina (Martinic, 1995). La medida con características proteccionista ocasionó trabas burocráticas al ser aplicada en la zona austral, perjudicando tanto a embarcaciones chilenas y argentinas que mantenían sus matrículas en el puerto de Punta Arenas. Para evitarlas, muchas embarcaciones trasladaron sus matrículas a puertos transandinos, lo que ocasionó una pérdida de ingresos para la ciudad y la entrada de nuevos actores en el transporte marítimo 


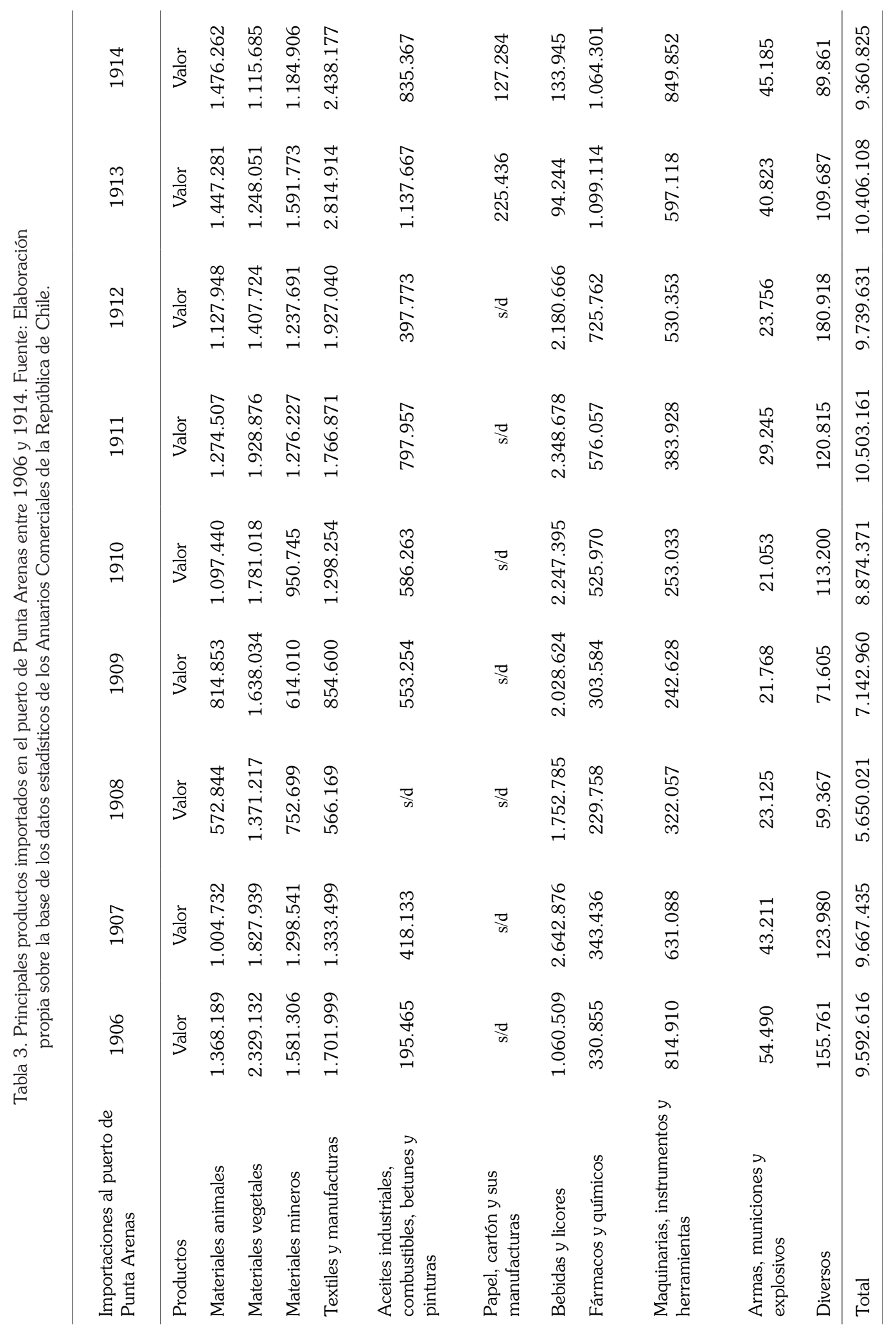


Tabla 4. Principales productos nacionales "importados" al puerto de Punta Arenas entre 1903 y 1906. Fuente: Elaboración propia sobre la base de los datos estadísticos del Censo General del Territorios de Magallanes de 1906.

\begin{tabular}{lcccc}
\hline & 1903 & 1904 & 1905 & 1906 \\
\hline Productos & Valor & Valor & Valor & Valor \\
\hline Suelas & 7.092 & 8.834 & 7.622 & 13.141 \\
Calzados surtidos & 30.563 & 40.318 & 55.637 & 37.905 \\
Cebollas & 4.230 & 5.170 & 6.873 & 11.174 \\
Descorazados & 4.552 & 6.290 & 4.698 & 2.410 \\
Frijoles & 24.846 & 18.298 & 19.921 & 36.694 \\
Frutas frescas & 8.687 & 18.254 & 14.344 & 32.430 \\
Legumbres frescas & 4.132 & 6.032 & 7.232 & 11.541 \\
Nueces & 3.085 & 6.394 & 2.734 & 3.886 \\
Papas & 64.436 & 80.737 & 86.981 & 150.499 \\
Pasas & 5.889 & 6.812 & 5.385 & 6.123 \\
Trigo & 5.576 & 6.280 & 6.049 & 6.138 \\
Frutas en conserva & 13.860 & 26.910 & 15.834 & 23.796 \\
Harina flor & 16.457 & 42.690 & 29.930 & 4.920 \\
Madera para construcción & 14.930 & 39.683 & 17.607 & 7.285 \\
Aguardiente & 6.610 & 4.375 & 1.600 & 700 \\
Cerveza & 5.521 & 9.600 & 18.502 & 22.332 \\
Chicha de uva & 4.673 & 10.357 & 6.721 & 4.502 \\
Vino tinto & 277.294 & 297.230 & 298.406 & 234.367 \\
Maquinas a vapor & 6.000 & - & - & - \\
Maquinas surtidas & 2.005 & - & - & 11.135 \\
\hline
\end{tabular}

provenientes de Buenos Aires, deteriorando la hegemonía de Punta Arenas sobre los territorios de Chubut, Santa Cruz y Tierra del Fuego, tanto argentina como chilena.

\section{Instalación de la aduana de Punta Arenas}

Tras cerca de dos décadas de lobby por parte del empresariado del centro-sur de Chile, en 1912 y con un arduo debate en el parlamento se aprueba la instalación de la aduana de Punta Arenas, poniendo fin al privilegio que mantenía la región de contar con puerto libre.

La instalación de la aduana fue principalmente impulsada por la Sociedad de Fomento Fabril que buscaba expandirse al mercado austral. Anteriormente la Sociedad había ejercido presión en tres oportunidades; en 1899 mediante la visita del presidente Federico Errázuriz quien declara la instalación de una aduana pero no dispuso de ninguna ley para respaldarla, por lo que se funda una aduana con la sola intención de recopilar datos estadísticos; en 1907 a través del presidente Pedro Montt que luego de escuchar las peticiones del empresariado, emprende un viaje a Punta Arenas donde encuentra inoportuna la instalación por los efectos negativos que generaría para la región; y en abril de 1912 donde mediante parlamento se hace efectivo el proyecto regularizando el puerto con la ley que impera en el resto del país.

Los argumentos utilizados en el Parlamento a favor de la instalación de la aduana fueron principalmente tres. El desarrollo de una "acción chilenizadora" mediante la apertura de nuevos productos nacionales a un mercado donde la 
presencia chilena se había mermado en desmedro de los productos europeos. La eliminación del contrabando existente en el centro de la región producido por el estado franco del puerto de Punta Arenas, el cual era aprovechado por comerciantes desleales para generar una competencia beneficiosa a ellos por adquisición de productos a menor costo por la inexistencia de aranceles; y por último, para aumentar las arcas fiscales mediante el cobro a las embarcaciones que utilizaban el puerto.

El Parlamento autorizó el destino de 200 mil pesos para los gastos que generaría la instalación de la aduana en Punta Arenas, la que tendría una inversión final de 160 mil y la cual durante el primer año de funcionamiento mantendría ingresos sobre los 300 mil pesos (Tabla 5). Sin embargo, la infraestructura instalada no fue la más adecuada para la zona, ya que a los problemas burocráticos generados por las nuevas exigencias y la inadecuada infraestructura que no daba abasto para los funcionarios públicos y la gente que utilizaba el servicio, se sumó la inexistencia de galpones para resguardar los bienes que necesitaban esperar en el puerto mientras se realizaban las labores burocráticas (Gómez, 1914). La situación generó que a la espera de la documentación necesaria tanto para importar como exportar, los productos eran puestos en custodia bajo ninguna protección y por lo tanto a las inclemencias del clima que terminaban por deteriorarlos.

Las demoras y los problemas administrativos que supuso la nueva administración aduanera, produjeron el desplazamiento del comercio hacia el puerto libre argentino de Rio Gallegos, sin embargo, su efecto fue paulatino producto de la falta de infraestructura portuaria en Rio Gallegos $y$ otros puertos argentinos que no podían abordar todo el tráfico marítimo que circulaba por la zona (Gómez, 1914).

Consecuencia anticipada de la discusión en el Parlamento de la instalación de la aduana en Punta Arenas, muchos comerciantes en la incertidumbre de las consecuencias de su aplicación, subieron en más de $45 \%$ los precios de los productos alimenticios a mediados de febrero. Sin embargo, el mayor golpe para la población de Magallanes fue la subida de un $30 \%$ en el precio de la leche condensada, bien
Tabla 5. Ingresos fiscales generados por la aduana de Punta Arenas desde su instalación en abril de 1912 a diciembre de 1912. Fuente: Datos obtenidos de tabla de diario "El

Magallanes”, edición del 3 de enero de 1913.

\begin{tabular}{cc}
\hline Desde 15 al 30 de Abril & $17.815,36$ \\
\hline Mayo & $38.342,94$ \\
Junio & $40.333,20$ \\
Julio & $34.641,95$ \\
Agosto & $33.784,25$ \\
Septiembre & $33.424,20$ \\
Octubre & $37.100,44$ \\
Noviembre & $40.887,05$ \\
Diciembre & $29.927,75$ \\
\hline Total & $305.755,14$ \\
\hline
\end{tabular}

de primera necesidad en muchos de los hogares magallánicos que contaban con recién nacidos o niños. La importancia de la leche condensada se da a raíz de las inclemencias del clima, la cual no hace viable la producción de leche de ternera, teniendo que recurrir a la importación de la leche enlatada (FOM, 1915).

La especulación en los precios antes de la aprobación de la aduana, había generado un descontento en la población que no veía un aumento salarial, el cual era fuertemente demandado por la Federación Obrera de Magallanes (FOM). El descontento llegó a la prensa de El Magallanes, que en su edición del sábado 17 de febrero de 1912 es publicado por "El Centro Oficios Varios" un artículo titulado "Trastorno Comercial provocado por el Impuesto aduanero":

En ocasión anterior habiamos hecho notar el alza prematura que el comercio en general había hecho experimentar en todos los artículos desde antes que se supiera de seguro la aprobación de la ley aduanera para Punta Arenas, cuando aún se discutía ducha ley en nuestras Cámaras. Esto Indicaba que existía cierto espíritu especulativo, cuya justificación no podemos aceptar y que ha venido a acentuarse más aun después que se tuvo conocimiento cabal de la total aprobación de dicha ley, pues han sido recargados por un treinta por ciento todos los artículos en general. 
Este recargo de treinta por ciento en artículos de primera necesidad y que no van a ser gravados con el impuesto, como el azúcar, el aceite, la harina, el arroz el té y el café, viene a comprobar que no hay justicia en los procedimientos dele comercio, aumentado con ello el clamor del público.

El pánico del primer momento pudo haber dado ya lugar a la reflexión y la calma inclinando a nuestro comercio a marchar con más cautela no haciendo sentir al público de un modo tan brusco y prematuro los efectos de una ley muy mal concebida sin duda, pero cuyas consecuencias no son tan desastrosas como se le creyó en el primer momento.

Consideramos ya oportuna
la intervención de la autoridad
administrativa, la que podría convocar
una reunión de comerciantes, en la que
tendría representación mayoristas y
minoristas, para estudiar la situación
grave que se presenta, como que ella afecta
directamente al bienestar del pueblo.

La primera huelga por causa del aumento de los precios se realizó entre el 29 de febrero al 4 de marzo de 1912, la cual contó con una amplia convocatoria que recorrió las calles y la plaza Muñoz Gamero con estandartes rojos. El relativo éxito de la huelga motivó a las organizaciones sociales regionales a exigir mejoras salariales y en las condiciones laborales que vivían gran parte de los trabajadores. Así fue que durante los primeros días de diciembre y justo al empezar las faenas de esquila se volvió a gestar una nueva huelga de carácter regional y que duraría hasta el 13 de diciembre tras la acogida de gran parte de las peticiones de los trabajadores. Como consecuencia de la huelga, pudo haber generado un ambiente de malestar entre los trabajadores de la región que desencadeno la baja en la exportación de lana durante el año 1912.

Construcción del Canal de Panamá en 1914 y la Primera Guerra Mundial

La construcción del canal de Panamá significó el acontecimiento externo más esperado durante la primera década del siglo XX y el que menos impacto tuvo a corto plazo. La instalación de la aduana y la ley de cabotaje argentino significaron golpes críticos para la región, la cual vio poco a poco perder la hegemonía de antaño. El efecto que tuvo la inauguración del canal de Panamá se vio opacado por el inicio de la Primera Guerra Mundial en Europa, la cual disminuyó notablemente el tráfico marítimo internacional que mantenía el puerto chileno. Durante el primer semestre de 1914 el tráfico al puerto de Punta Arenas fue de 152 naves, descendiendo a 47 durante el primer semestre de 1915 y llegando a prácticamente 0 para el segundo semestre de 1918 (Martinic, 2006b). Fue recién hacia el 1919 cuando se asumió que los barcos mercantes europeos dejarían de aparecer por las costas australes (Entrevista de Prensa El Pingüino a Mateo Martinic, 4 de julio 2016).

La decadencia del tráfico además de cortar el vínculo de Magallanes con Europa, cortó el movimiento con los puertos del Atlántico y Pacífico. Las compañías navieras provenientes de Europa eran las que ofrecían el servicio de transporte hacia los puertos del resto de América, sin su presencia no pudo cubrirse la frecuencia de las embarcaciones.

Además, finalizada la Gran Guerra, el escenario internacional seguía sin favorecer a la región, ya que el comercio marítimo de las potencias europeas demoró años en recuperar los niveles del período preguerra, si es que realmente logró recuperarse (Martinic, 2012). El tráfico de cabotaje regional que significaba el $10 \%$ del movimiento en la región, fue decayendo levemente. Sin embargo, en los años siguientes al conflicto mundial, el puerto de Punta Arenas todavía ostentaba una influencia a nivel regional, principalmente enfocada en la exportación lanar, sus derivados de carne frigorizada y sus subproductos, los cuales cubrían la salida del $60 \%$ del total producido en ambos lados de la Patagonia como indica el estudio Pioneer Settlement. Cooperative Studies by twenty-six authors de 1932 (Joerg, 1932). No obstante los tiempos donde se apreciaba la presencia de tres o cuatro embarcaciones diarias en el puerto chileno quedaban atrás, y con él la época dorada de Punta Arenas. 


\section{CONCLUSIÓN}

El puerto de Punta Arenas significó un polo de desarrollo para toda la región austral y punto de apoyo seguro para la navegación transoceánica. Su desarrollo no hubiera sido posible sin la creación de una robusta economía basada en la lana de oveja y sus derivados, los cuales demandaban los mercados europeos, principalmente en el Imperio Británico e Imperio Alemán. La amplia historiografía regional cubre con claridad este período, el cual se cataloga como de auge gracias al desarrollo alcanzado por la ciudad. Sin embargo y con fuentes inéditas, este estudio otorga una nueva faceta al incorporar datos que solamente eran consultados de forma general.

La información recabada complementa lo ya desarrollado hasta ahora, validando el auge comercial del puerto con aproximación estadística, demostrando la importancia para el comercio internacional de la producción de ovino y sus derivados tras la instalación de los primeros frigoríficos. Las estadísticas muestran el crecimiento experimentado por el tráfico comercial por el estrecho de Magallanes, el cual tuvo su mayor apogeo en tonelaje de entrada en 1910 pero en valor comercial en 1911 pero luego con una abrupta caída al año siguiente. Mientras que el mayor valor de exportación alcanzado por los principales productos elaborados en la región se ubicó en 1914 con un total de 26.309.669 pesos. Sin embargo y principalmente el impacto que ocasiona la instalación de la aduana y la ley de cabotaje argentino, inició el declive de un período que terminaría con la Primera Guerra Mundial y posteriormente con el efecto a largo plazo de la apertura del canal de Panamá.

\section{BIBLIOGRAFÍA}

Bascuñán C., Eichholz M., \& Hartwig, F. (2013) Naufragios en el Océano Pacífico Sur. Tomo 1. Editorial Tauru.
Ciselli, G. (1999). Un Espacio Compartido al Sur del Paralelo 46 (1880-1944). II Congreso de Historia Social y Política.

Gomez, A. (1914). Viaje de un chileno a Magallanes. Santiago de Chile: Ediciones Universitaria.

Joerg, W. (1932) Settlement. Cooperative Studies by twenty authors. American Geographical Society Special Publication, 14.

Martinic, M. (1977). Historia del Estrecho de Magallanes. Santiago de Chile: Edición Andrés Bello.

Martinic, M. (1995). Los Armadores de Punta Arenas de 1870 a 1930 . Revista Marina, 8.

Martinic, M. (2002a). Breve Historia de Magallanes. Ediciones de la Universidad de Magallanes.

Martinic, M. (2002b). La Participación de Capitales Británicos en el Desarrollo Económico del Territorio de Magallanes (1880-1920). Historia, 35, 299-321.

Martinic, M. (2006a). Historia de la Región Magallánica, Tomo II. Punta Arenas: Ediciones de la Universidad de Magallanes.

Martinic, M. (2006b). Historia de la Región Magallánica, Tomo III. Punta Arenas: Ediciones de la Universidad de Magallanes.

Martinic, M. (2012) Punta Arenas del Siglo XX. Punta Arenas: Edición Geopark.

Navarro, L. (1908). Censo Jeneral de Población i Edificación, Industria, Ganadería i Minería del Territorio de Magallanes, II Tomo.

Veksler, B. (2014). Artículo en Diario del Fin del Mundo "El 10 de Junio de 1908 Menéndez y Braun Deciden Fusionar Actividades Comerciales".

\section{Otras fuentes}

Anuario Comercial de la República de Chile de 1905 - 1913. Carta de Oscar Vial, 1870

Prensa Magallanes 1906-1910.

Entrevista de la Prensa El Pingüino a Mateo Martinic, 4 de julio 2016. 